\title{
Application of Geological Radar Method in Exploration of Reservoir Complex Pipelines
}

\author{
Jun YAN ${ }^{\mathrm{a}}$, Aiyu QU ${ }^{\mathrm{b}, 1}$, Hong CAI ${ }^{\mathrm{a}}$, Dingsong XIE ${ }^{\mathrm{a}}$ and Shuaifeng WU ${ }^{\mathrm{a}}$ \\ ${ }^{a}$ State Key Laboratory of Simulation and Regulation of Water Cycle in River Basin, \\ China Institute of Water Resources and Hydropower Research, Beijing 100048, China \\ ${ }^{\mathrm{b}}$ Chinese Academy of Environmental Planning, Beijing 100048, China
}

\begin{abstract}
Geodesic radar method is a non-destructive geophysical method to detect underground geological structure based on the differences in conductivity, magnetism and dielectric constants of different substances. This method has the advantages of economy, fast, continuous, accurate, non-destructive, non-contact, etc. It is applicable to both metal and non-metal pipelines, and it is the preferred tool for underground pipeline detection. In this paper, the geological radar method is used to detect the complex pipe network around Daning Reservoir in detail by laying out multiple detection lines. It provides basic support for the later pipe network rectification. The research results can provide experience for the detection of the complex pipe network in the urban reservoir.
\end{abstract}

Keywords. Geological radar method, underground geological structure, complex pipe network

\section{Introduction}

In recent years, with the construction of urban reservoirs, there are more residential, plant and other facilities around the reservoir, resulting in the embankment, through the dam water supply and drainage, gas, cables and other pipes dense, forming a more complex underground pipe network. However, due to incomplete clean-up, insufficient construction scale, lack of data preservation, inadequate daily maintenance and other issues, resulting in the underground pipe network "old", "secret", "disorder" situation is becoming more and more serious. Pipeline leakage, heavy rain and flooding, third-party construction and excavation of pipelines and other accidents in the city reservoir has appeared, seriously affecting the normal functioning of reservoir efficiency, but also threaten the safety of people's lives and property around the reservoir. Because the material, specification and transmission material of underground pipeline are different, and there are obvious differences between its physical properties and surrounding medium, it has become an effective technique to detect underground pipeline by geophysical method, especially the most common application of ground-seeking radar method.

Geodesic radar method is a non-destructive geophysical method to detect underground geological structure based on the differences in conductivity, magnetism and dielectric constants of different substances [1-3]. This method has the advantages [4] of

\footnotetext{
${ }^{1}$ Corresponding Author, Aiyu QU, Chinese Academy of Environmental Planning, Beijing 100048, China; Email: yanjunwuhan@163.com.
} 
economy, fast, continuous, accurate, non-destructive, non-contact, etc. It is applicable to both metal and non-metal pipelines, and it is the preferred tool for underground pipeline detection. Jiao et al. [5] used ground penetrating radar to accurately find out the location of underground pipelines and obstacles. The problems encountered in the process of underground pipeline detection and pipeline construction in Shenyang were successfully solved, ensuring the smooth construction and avoiding unnecessary economic losses. Zhao et al. [6] used ground-finding radar to detect some non-metallic water and gas pipelines in Jiaxing. Its high detection accuracy was verified by excavation. Xiong et al. [7] used the ground-seeking radar method to detect the flat position of gas pipelines, buried depth, inflection point and three-way points were studied, and pointed out that when using ground-finding radar to detect PE pipes, the ratio of pipe diameter to buried depth is greater than 0.1 is a more appropriate detection range. Li et al. [8] conducted positive simulation and field tests and pointed out that the PVC pipeline wrapped in tin foil and bound wire can effectively improve the detection effect, for ground radar detection deep buried nonmetallic pipeline provides a new way of thinking. Tian [9] analyzed the effect of groundfinding radar method to detect passing pipelines in Guangzhou Development Zone, and the results show that the method is obviously recognized for most underground pipelines, but its accuracy is affected by the size of the target pipe diameter, surrounding interference and terrain. Zheng et al. [10] used the Swedish MALA geodesic radar detector to check a sewage treatment plant, the results are consistent with the actual pipe location, no dark pipes and dark ditches were found. Ganiyu et al. [11] used ground-finding radar to detect sewage and water pipelines on the campus of the Federal Agricultural University of Abekuta in southern Nizhny, and the results were very relevant to the measured depth of excavation, with higher accuracy of the comparative resistivity method.

In this paper, the geological radar method is used to detect the complex pipe network around Daning Reservoir in detail by laying out multiple detection lines. It provides basic support for the later pipe network rectification. The research results can provide experience for the detection of the complex pipe network in the urban reservoir.

\section{Engineering Background}

The Daning Reservoir is located at east of Yongding River west adjacent to Changxindian Town, with a total storage capacity of 36 million $\mathrm{m} 3$ the engineering grades II. The main dam, sub-dam, floodgates, east embankment and west embankment are 2-level buildings. Since its completion, the pipeline distribution in the Daning reservoir area is huge. Since its completion, it has undergone many alterations and expansions, coupled with different management departments, which makes the pipeline statistics in the reservoir area more complex, and the location and geometry of most of the pipes are unknown.

\section{Detection Methods and Equipment}

Geo-radar method transmits high-frequency electromagnetic waves to the ground by continuous drag of antennas, and the electromagnetic wave signal reflects, transmits and refracts when it encounters a medium interface with electrical differences (such as differences in dielectric constants) when it travels within an object. The greater the difference in dielectric constants of the media on both sides of the interface, the greater 
the reflected electromagnetic wave energy, the greater the reflected electromagnetic wave is received by the receiving antenna that moves in sync with the transmitting antenna. The movement characteristics of the reflected electromagnetic wave are accurately recorded by the radar host, and the cross-sectional scanning radar image of the underground medium is obtained, and the purpose of identifying underground target objects is achieved through the processing and image interpretation of radar images.

The propagation velocity $V$ of electromagnetic waves in the uniform medium is constant. According to the time difference between the ground reflected wave on the geological radar record and the underground reflected wave, the buried depth of the underground anomaly $H$ can be derived according to the following formula:

$$
H=V \cdot \Delta T / 2
$$

where $H$ is the depth of the target layer; $V$ is the velocity at which electromagnetic waves travel through underground media, and their size is represented by the following:

$$
V=C / \sqrt{\varepsilon}
$$

where $C$ is the rate at which electromagnetic waves travel in the atmosphere, about $3 \times 10^{8}$ $\mathrm{m} / \mathrm{s} ; \varepsilon$ is a relative dielectric constant, depending on the dielectric constant of the material that forms the subsurface layers.

The amplitude of the radar wave reflected signal is proportional to the reflection coefficient. In a low-loss medium dominated by displacement current, the reflection coefficient $r$ can be expressed as:

$$
r=\frac{\sqrt{\varepsilon 1}-\sqrt{\varepsilon 2}}{\sqrt{\varepsilon 1}+\sqrt{\varepsilon 2}}
$$

where $\varepsilon 1, \varepsilon 2$ are the relative dielectric constant of the upper and lower media of the interface.

The intensity of the reflected signal mainly depends on the electrical difference between the upper and lower media. The greater the electrical difference, the stronger the reflected signal. The penetration depth of radar waves mainly depends on the electrical properties and center frequency of the underground medium. The higher the conductivity, the smaller the penetration depth is. The higher the center frequency, the smaller the penetration depth is.

The instrument used in this geological radar test is the Canadian Sensors and Software Pulse EKKO PRO professional geodesic radar. The main performance parameters of the equipment include:1) Host: data acquisition mode: point measurement, continuous measurement, range gauge control measurement, trigger mode: time trigger, keyboard trigger, range wheel trigger, marker trigger; A/D conversion: 16 bits; pulse frequency: 200 $\mathrm{kHz}$; measurement window: 5 to $5000 \mathrm{~ns}$; system power consumption:15 W; maximum dynamic range: $156 \mathrm{~dB}$; /b120> $300 \mathrm{~V}(200 \mathrm{MHz}$ antenna); 2) Antenna: Antenna Type: $100 \mathrm{MHz}$ dual-shot dual-receiving antenna. 


\section{Probe Scheme Design}

In this pipeline network detection, 4 radar survey lines were arranged at the corresponding positions of the main dam, auxiliary dam, east embankment, and west embankment of the reservoir. Line layout is shown in figure 1. Figure 2 shows the on-site detection situation.

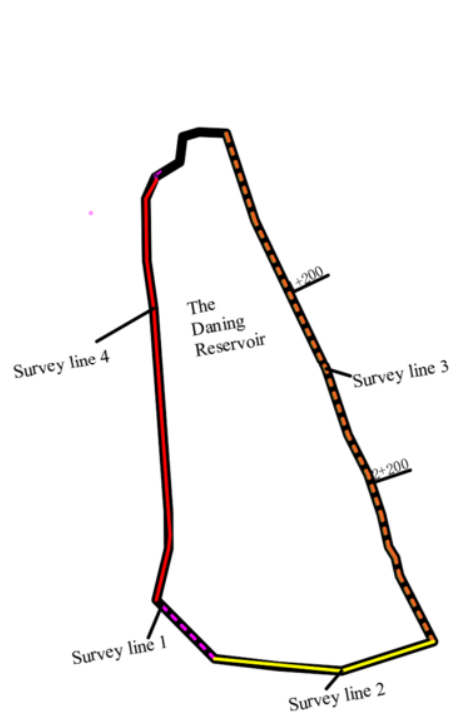

Figure 1. The arrangement of the geological radar detection line of the reservoir dam.
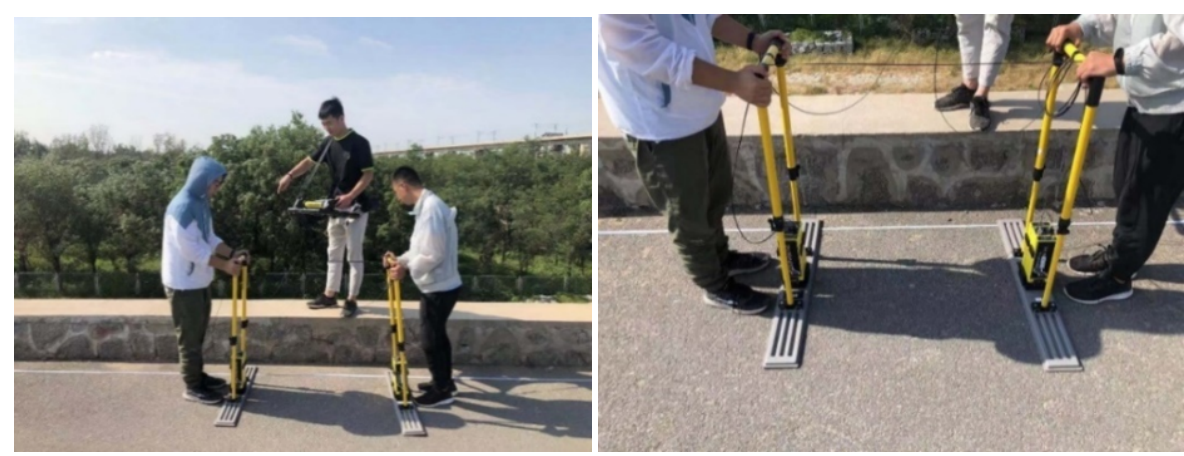

Figure 2. Geological radar detection site detection.

\section{Analysis of Detection Results}

A total of 11 pipelines were found within the range detected by this geological radar, and typical pipeline detection results are shown in figures 3-6 below. 


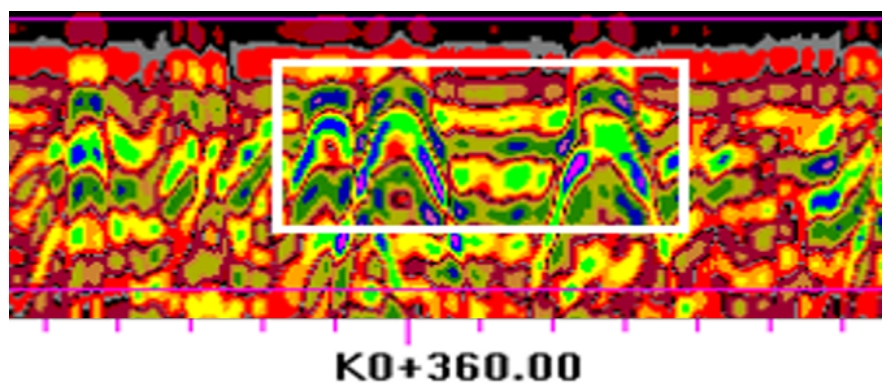

Figure 3. Sub-dam line k0-363.8 (pipeline).

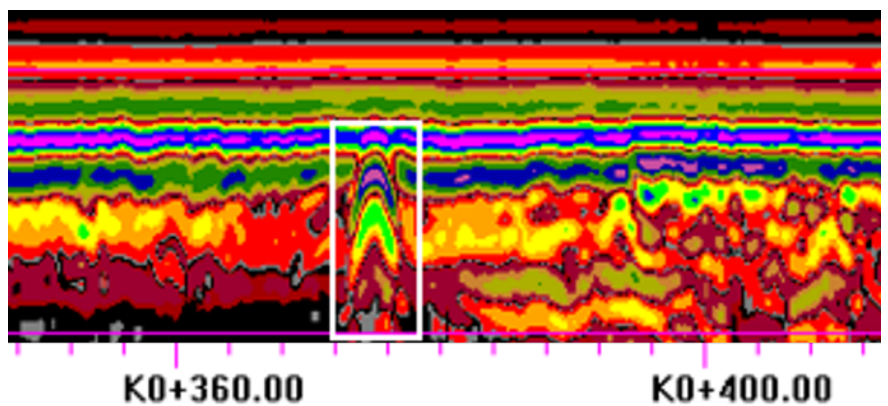

Figure 4. Sub-dam measuring line k0-375.4 (pipeline).

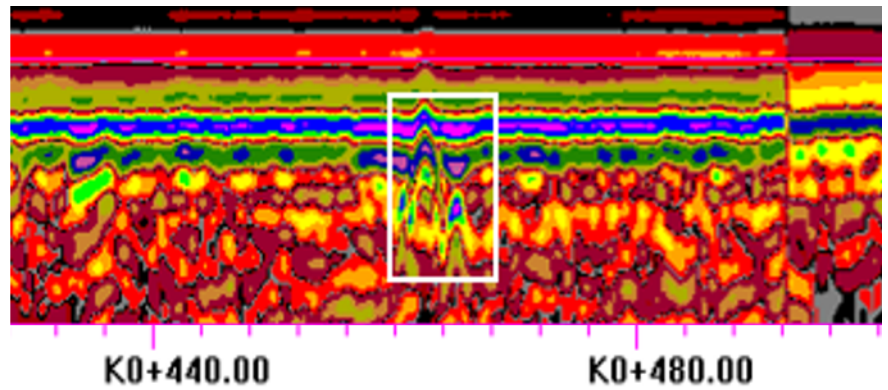

Figure 5. Sub-dam line k0-462.5 (pipeline). 


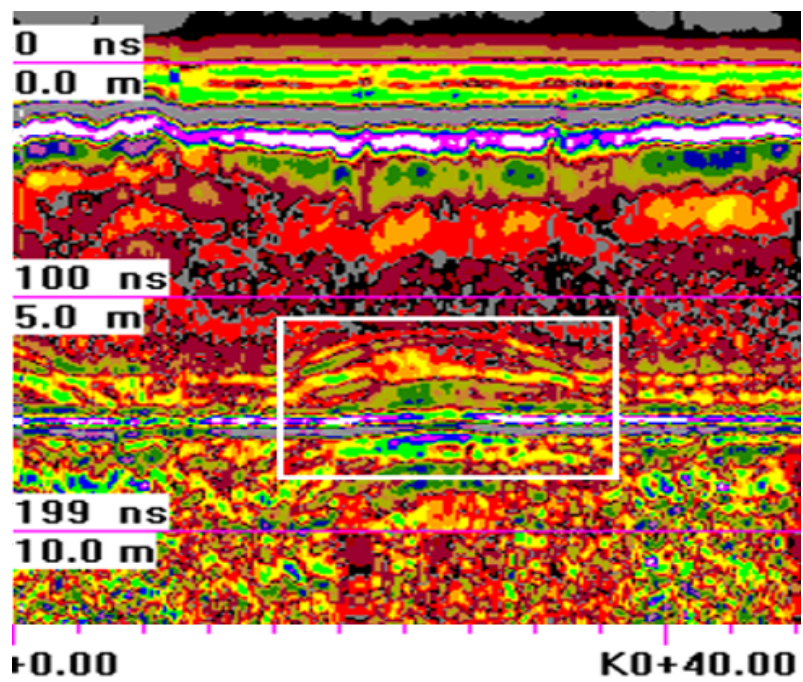

Figure 6. West Embankment Line k0-25.3 (suspected pipeline).

According to the results of pipeline exploration, the distribution of the complex pipe network through the dam of Daning Reservoir is basically clarified, as shown in figure 7.

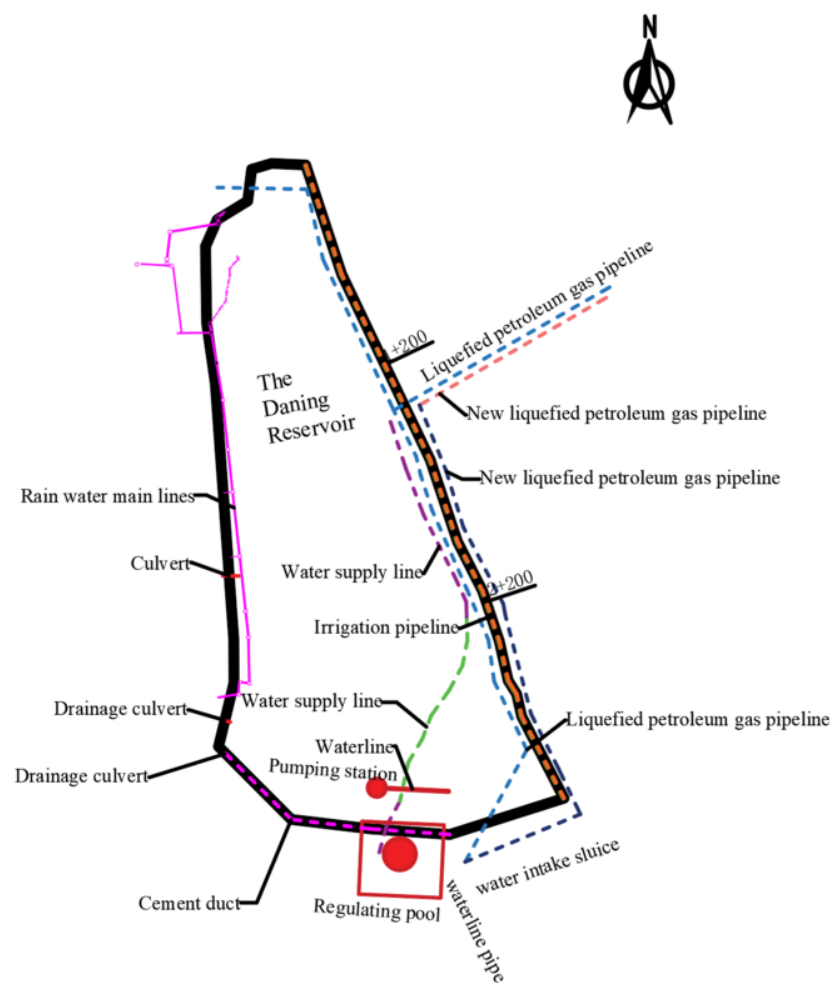

Figure 7. Underground pipe network exploration results. 


\section{Conclusions}

The water supply and drainage, gas, cable and other pipes around the city reservoir are dense, forming a more complex underground pipe network. Pipeline leakage, heavy rain and flooding, third-party construction and excavation of pipelines and other accidents in the city reservoir has appeared, seriously affecting the normal functioning of reservoir efficiency, but also threaten the safety of people's lives and property around the reservoir.

- The geological radar method is used to detect the complex pipe network around Daning Reservoir in detail by laying out multiple detection lines. It provides basic support for the later pipe network rectification;

- Geodesic radar method is a non-destructive geophysical method to detect underground geological structure based on the differences in conductivity, magnetism and dielectric constants of different substances. It is applicable to both metal and non-metal pipelines, and it is the preferred tool for underground pipeline detection.

\section{Acknowledgments}

This research was financially supported by the National Key Technologies Research \& Development Program (2017YFC0804600), High-level Innovative Talents Project of Guizhou Province, China (No. 2018-5630) and the IWHR Research \& Development Support Program (GE0145B562017).

\section{References}

[1] Xu X L, Zhao Y L, Wang F and Wang W J 2012 Review on diameter detection of underground pipe with GPR Progress in Geophysics 27 (5) 206-2215.

[2] Zeng Y M, Jia X X and Li Y J 2016 Buried PE pipeline positioning detection technology overview Mapping Advisory (S1) 64-88. (in Chinese)

[3] Zhang J L 2019 Application research on construction technology of trenchless directional drilling Policy Research \& Exploration (01) 30-31.

[4] Jol H 2009 Ground Penetrating Radar: Theory and Applications (Amsterdam: Elsevier).

[5] Jiao J, Qu S B and Yang R J 2006 Application of ground penetrating radar technology in detecting underground objects in Shenyang Geotechnical 9 (10) 79-80. (in Chinese)

[6] Zhao Y F 2009 Application of ground penetrating radar in non-metallic pipeline detection in Jiaxing City Municipal Technology 27 (3) 307-323. (in Chinese)

[7] Xiong J N, Sun M, Peng C, Wang Z G, Liu S and Zhou J 2017 Urban gas PE pipeline detection method based on ground-finding radar Geophysical and Geochemical Exploration 39 (5) 1079-1084. (in Chinese)

[8] Li X D and Wang Y Q 2016 Buried PVC pipeline geological radar detection method improved Modern Mining 32 (4) 270-271. (in Chinese)

[9] Tian F 2017 Analysis on practical detection methods of underground non-metallic pipelines Land and Resources of North China (05) 103-105. (in Chinese)

[10] Zheng L L and Qin Z 2018 Application of ground penetrating radar in city sewage pipeline detection Journal of Huainan Vocational and Technical College 18 (5) 5-6. (in Chinese)

[11] Ganiyu S A, Oladunjoye M A, Onakoya O I, Olutoki J O and Badmus B S 2020 Combined electrical resistivity imaging and ground penetrating radar study for detection of buried utilities in Federal University of Agriculture, Abeokuta, Nigeria Environ. Earth Sci. 79 (8) 177. 